J. Korean Math. Soc. 51 (2014), No. 5, pp. 941-953

http://dx.doi.org/10.4134/JKMS.2014.51.5.941

\title{
SPECTRAL RADIUS OF BIORTHOGONAL WAVELETS WITH ITS APPLICATION
}

\author{
Qingyun Zou, Guoqiu Wang, And Mengyun Yang
}

\begin{abstract}
In this paper, a 2-circular matrix theory is developed, and a concept of spectral radius for biorthogonal wavelet is introduced. We propose a novel design method by minimizing the spectral radius and obtain a wavelet which has better performance than the famous 9-7 wavelet in terms of image compression coding.
\end{abstract}

\section{Introduction}

Over the last two decades, wavelets have become a fundamental tool in many areas of applied mathematics and engineering ranging from signal and image processing to numerical analysis. In her celebrated paper [2], Daubechies introduced a general method to construct compactly supported wavelets. It is well known that 2-band orthogonal wavelet suffers from severe constraint conditions. For instance, nontrivial symmetric 2-band orthogonal wavelet does not exist except the Haar wavelet [3]. Biorthogonal wavelet enjoy two important properties of linear phase and higher vanishing moments, was studied $[4,8,11,12]$. Vetterli and Herley studied theory and design for biorthogonal wavelet [9] and Cohen gave another biorthogonal wavelet family [6]. Guoqiu Wang constructed wavelet filters with free paraments based on 2-circular matrix method [1]. Even though the research on biorthogonal wavelet is relatively mature, further study is still valuable.

Let $(\psi, \widetilde{\psi})$ be a pair of dual wavelet functions,

$$
\begin{aligned}
& \psi_{j, k}(x)=2^{j / 2} \psi\left(2^{j / 2} x-k\right), j, k \in \mathbb{Z}, \\
& \widetilde{\psi}_{j, k}(x)=2^{j / 2} \widetilde{\psi}\left(2^{j / 2} x-k\right), j, k \in \mathbb{Z} .
\end{aligned}
$$

Received June 27, 2013; Revised April 6, 2014.

2010 Mathematics Subject Classification. 42C40, $42 \mathrm{~A} 16$.

Key words and phrases. 2-circular matrix, biorthogonality, vanishing moments, filters of even lengths, image compression.

This work was supported by the National Science Foundation of China (Grant No. 11171103), the Science Foundation of Hunan Education Office (13C624), the Science Foundation of Hunan University of Arts and Science (13ZD01).

(C)2014 Korean Mathematical Society 
Here $\left\{\psi_{j, k}\right\}$ and $\left\{\widetilde{\psi}_{j, k}\right\}$ constitute wavelet frames, respectively, if there exist positive real numbers $A, B, \widetilde{A}$ and $\widetilde{B}$ such that

$$
\begin{aligned}
& A\|f\|^{2} \leq \sum_{j, k}\left|\left\langle f, \psi_{j, k}(x)\right\rangle\right|^{2} \leq B\|f\|^{2}, \\
& \widetilde{A}\|f\|^{2} \leq \sum_{j, k}\left|\left\langle f, \widetilde{\psi}_{j, k}(x)\right\rangle\right|^{2} \leq \widetilde{B}\|f\|^{2}
\end{aligned}
$$

for all $f \in L^{2}(R)$.

(1.3) and (1.4) show that the energy of biorthogonal wavelet transform is controllable although it is not conservative. However, while $B-A$ or $\widetilde{B}-\widetilde{A}$ are rather large, the biorthogonal wavelet transform may be unstable. i.e., the energy is amplified in some cases and compressed in other cases.

In view of potential applications, estimating the bounds of sub-band operator could be more important. Let's recall the sub-band coding scheme or Mallat algorithm associated to a biorthogonal wavelet. There are four sequences $h=$ $\left(h_{n}\right)_{n \in Z}, g=\left(g_{n}\right)_{n \in Z}, \widetilde{h}=\left(\widetilde{h}_{n}\right)_{n \in Z}, \widetilde{g}=\left(\widetilde{g}_{n}\right)_{n \in Z}$, two of which are used for decomposition $\{h, g\}$ and two for reconstruction $\{\widetilde{h}, \widetilde{g}\}$. Starting from a data sequence $c^{0}=\left(c_{n}^{0}\right)_{n \in Z}$, we convolve with $h, g$ and retain only one sample out of every two for the decomposition:

$$
\begin{aligned}
& c_{n}^{1}=\sum_{k} h_{2 n-k} c_{k}^{0}, \\
& d_{n}^{1}=\sum_{k} g_{2 n-k} c_{k}^{0} .
\end{aligned}
$$

The reconstruction operation is

$$
c_{k}^{0}=\sum_{n}\left(\widetilde{h}_{2 n-k} c_{n}^{1}+\widetilde{g}_{2 n-k} d_{n}^{1}\right) .
$$

(1.5) and (1.6) can be rewritten as the form of 2-circular matrix [10], which can be defined by the 2-circular operator. Let $v=\left(v_{1}, v_{2}, v_{3}, \ldots, v_{n}\right)$. Define

$$
\sigma^{0}(v)=v, \sigma(v)=\left(v_{n-1}, v_{n}, v_{1}, v_{2}, v_{3}, \ldots, v_{n-2}\right), \sigma^{k}(v)=\sigma^{k-1}(\sigma(v)) .
$$

If $v=\left(h_{0}, h_{1}, h_{2}, \ldots, h_{p}, 0, \ldots, 0, h_{-p+1}, \ldots, h_{-1}\right)$ and $u=\left(g_{0}, g_{1}, g_{2}, \ldots, g_{q}, 0\right.$, $\left.\ldots, 0, g_{-q+1}, \ldots, g_{-1}\right)$ are two $2 n$-dimensional row vectors, then

$$
M_{2 n}=\left[\begin{array}{c}
\sigma^{0}(v) \\
\sigma^{1}(v) \\
\vdots \\
\sigma^{n-1}(v) \\
\sigma^{0}(u) \\
\sigma^{1}(u) \\
\vdots \\
\sigma^{n-1}(u)
\end{array}\right]
$$


is called the 2-circular matrix generated by $\{h, g\}$. An example is as follows:

$$
M_{8}=\left[\begin{array}{cccccccc}
h_{0} & h_{1} & h_{2} & h_{3} & 0 & 0 & h_{-2} & h_{-1} \\
h_{-2} & h_{-1} & h_{0} & h_{1} & h_{2} & h_{3} & 0 & 0 \\
0 & 0 & h_{-2} & h_{-1} & h_{0} & h_{1} & h_{2} & h_{3} \\
h_{2} & h_{3} & 0 & 0 & h_{-2} & h_{-1} & h_{0} & h_{1} \\
g_{0} & g_{1} & g_{2} & g_{3} & 0 & 0 & g_{-2} & g_{-1} \\
g_{-2} & g_{-1} & g_{0} & g_{1} & g_{2} & g_{3} & 0 & 0 \\
0 & 0 & g_{-2} & g_{-1} & g_{0} & g_{1} & g_{2} & g_{3} \\
g_{2} & g_{3} & 0 & 0 & g_{-2} & g_{-1} & g_{0} & g_{1}
\end{array}\right] .
$$

If $c^{0}$ is a periodic signal, we rewrite $c^{0}=\left(c_{1}^{0}, c_{2}^{0}, \ldots, c_{2 n}^{0}\right)^{T}$, which is a whole period.

Let $c^{1}=\left(c_{1}^{1}, c_{2}^{1}, \ldots, c_{n}^{1}, d_{1}^{1}, d_{2}^{1}, \ldots, d_{n}^{1}\right)^{T}$. Then there exists a $2 n \times 2 n 2$ circular matrix $M_{2 n}$ generated by $\{h, g\}$ such that

$$
c^{1}=M_{2 n} c^{0} \text {. }
$$

It is easy to see that (1.7) is equivalent to (1.5). Let

$$
c^{0}=\widetilde{M}_{2 n}^{T} c^{1},
$$

where $\widetilde{M}_{2 n}$ is a 2 -circular $2 n \times 2 n$ matrix generated by $\{\widetilde{h}, \widetilde{g}\}$.

Clearly, (1.8) is equivalent to (1.6). Then,

$$
\left\|c^{1}\right\|^{2}=\left(c^{1}\right)^{T} c^{1}=\left(c^{0}\right)^{T}\left(M_{2 n}^{T} M_{2 n}\right) c^{0} .
$$

Since $M_{2 n}^{T} M_{2 n}$ is a positive definite matrix, its eigenvalues $\lambda_{i}(i=1,2 \ldots, 2 n)$ are positive and there exists an orthonormal matrix $Q$ such that

$$
M_{2 n}^{T} M_{2 n}=Q^{T} \operatorname{diag}\left(\lambda_{1}, \lambda_{2}, \ldots, \lambda_{2 n}\right) Q .
$$

Let $s=Q c^{0}=\left(s_{1}, s_{2}, \ldots, s_{2 n}\right)^{T}$. Then $\|s\|^{2}=\left\|c^{0}\right\|^{2}$. It follows from (1.9) and (1.10) that

$$
\left\|c^{1}\right\|^{2}=\left(Q c^{0}\right)^{T} \operatorname{diag}\left(\lambda_{1}, \lambda_{2}, \ldots, \lambda_{2 n}\right)\left(Q c^{0}\right)=\sum_{i=1}^{2 n} \lambda_{i} s_{i}^{2} .
$$

Thus,

$$
\min \left\{\lambda_{i}\right\}\left\|c^{0}\right\|^{2} \leq\left\|c^{1}\right\|^{2} \leq \max \left\{\lambda_{i}\right\}\left\|c^{0}\right\|^{2}
$$

Similarly,

$$
\min \left\{\widetilde{\lambda}_{i}\right\}\left\|c^{0}\right\|^{2} \leq\left\|c^{1}\right\|^{2} \leq \max \left\{\widetilde{\lambda}_{i}\right\}\left\|c^{0}\right\|^{2},
$$

where $\widetilde{\lambda}_{i}(i=1,2 \ldots, 2 n)$ are the eigenvalues of $\widetilde{M}_{2 n}^{T} \widetilde{M}_{2 n}$.

Instead of estimating the bounds in (1.3) or (1.4), we try to calculate the eigenvalues of $M_{2 n}^{T} M_{2 n}$ or $\widetilde{M}_{2 n}^{T} \widetilde{M}_{2 n}$. It has been shown that the eigenvalues of $M_{2 n}^{T} M_{2 n}$ appear in pairs of reciprocal, $M_{2 n}^{T} M_{2 n}$ and $\widetilde{M}_{2 n}^{T} \widetilde{M}_{2 n}$ have the same eigenvalues (cf. [12, Section 2]). It is obvious that $\max \left\{\lambda_{i}\right\}=\max \left\{\widetilde{\lambda}_{i}\right\}=$ $\frac{1}{\min \left\{\lambda_{i}\right\}}=\frac{1}{\min \left\{\tilde{\lambda}_{i}\right\}}$. 
Note that $c^{0}$ only has finite length in (1.11). If $c^{0} \in l^{2}$, we shall investigate the $\operatorname{limit}_{n \rightarrow+\infty} \max \left\{\lambda_{i}\right\}$ to directly evaluate or examine the performances of filters, which is called the spectral radius of the biorthogonal wavelet in this paper.

This paper is organized as follows. In Section 2, we develop some results of the transform matrix, define the concept of spectral radius and prove the existence and uniqueness. An example is provided to illustrate our results in this paper. In Section 3, we propose a novel design method for constructing biorthogonal wavelets and obtain a wavelet with better performance of image compression. Conclusions are summarized in Section 4.

\section{Spectral radius of biorthogonal wavelet}

Assume that the low pass filters are FIR and symmetric. In addition, we assume

$$
\sum_{k} h_{k}=\sum_{k} \widetilde{h}_{k}=\sqrt{2}
$$

and let

$$
\begin{aligned}
& g_{k}=(-1)^{(1-k)} \widetilde{h}_{1-k}, \\
& \widetilde{g}_{k}=(-1)^{(1-k)} h_{1-k} .
\end{aligned}
$$

Then

$$
\begin{aligned}
& \sum_{k} h_{2 k}=\sum_{k} h_{2 k+1}=\frac{\sqrt{2}}{2}, \\
& \sum_{k} \widetilde{h}_{2 k}=\sum_{k} \widetilde{h}_{2 k+1}=\frac{\sqrt{2}}{2} .
\end{aligned}
$$

The perfect reconstruction condition is equivalent to the following equation

$$
\sum_{k} h_{k} \widetilde{h}_{k+2 j}=\delta_{j}
$$

where $\delta_{j}$ is the Dirac sequence, i.e., $\delta_{j}=1$ for $j=0$ otherwise $\delta_{j}=0$.

Define

$$
b_{k}=\sum_{i} h_{i} h_{i+2 k}, \quad \widetilde{b}_{k}=\sum_{i} \widetilde{h}_{i} \widetilde{h}_{i+2 k}
$$

Since $h$ and $\widetilde{h}$ are the FIR filters, let $d_{1}, d_{2}$ be the maximal positive integers such that $b_{d_{1}}=\sum_{i} h_{i} h_{i+2 d_{1}} \neq 0$ and $\widetilde{b}_{d_{2}}=\sum_{i} \widetilde{h}_{i} \widetilde{h}_{i+2 d_{2}} \neq 0$, respectively.

Define

$$
\begin{gathered}
u_{n, j}=\left(b_{0}+\widetilde{b}_{0}\right)+2 \sum_{i=1}^{d}\left(b_{i}+\widetilde{b}_{i}\right) \cos \left(\frac{2 \pi i j}{n}\right) 1 \leq j \leq\left[\frac{n}{2}\right], \\
u_{0}=\left(\left(b_{0}+\widetilde{b}_{0}\right)+2 \sum_{i=1}^{d}(-1)^{i}\left(b_{i}+\widetilde{b}_{i}\right)\right),
\end{gathered}
$$

where $d=\max \left\{d_{1}, d_{2}\right\}$ and $[x]$ denotes the largest integer less than or equal to $x$. 
We first present our main theorem of this paper based on a 2-circular matrix theory.

Theorem 2.1. Assume that $M_{2 n}=\left[{ }_{G}^{H}\right]$ is a 2-circular matrix generated by $\{h, g\}$, where $H$ and $G$ are $n \times 2 n$ 2-circular matrices. Then the characteristic polynomial of $M_{2 n} M_{2 n}^{T}$ is

$$
p(\lambda)= \begin{cases}(\lambda-1)^{2} \prod_{j=1}^{r}\left(\lambda^{2}-u_{n, j} \lambda+1\right)^{2} & n=2 r+1 ; \\ (\lambda-1)^{2}\left(\lambda^{2}-u_{0} \lambda+1\right) \prod_{j=1}^{r}\left(\lambda^{2}-u_{n, j} \lambda+1\right)^{2} & n=2 r+2,\end{cases}
$$

where $\omega=\cos \left(\frac{2 \pi}{n}\right)+i \sin \left(\frac{2 \pi}{n}\right), i$ is the imaginary unit.

To prove Theorem 2.1, we need some results as follows.

\section{Proposition 2.2.}

$$
\sum_{k} b_{k}=1, \quad \sum_{k} \widetilde{b}_{k}=1
$$

where $b_{k}$ and $\widetilde{b}_{k}$ are defined in (2.5).

Proof.

$$
\begin{aligned}
\sum_{k} b_{k} & =\sum_{k} \sum_{i} h_{i} h_{i+2 k}=\sum_{i} h_{i}^{2}+\sum_{k>0} \sum_{i} h_{i} h_{i+2 k}+\sum_{k<0} \sum_{i} h_{i} h_{i+2 k} \\
& =\sum_{i \in 2 Z+1} h_{i}^{2}+2 \sum_{k>0} \sum_{i \in 2 Z+1} h_{i} h_{i+2 k}+\sum_{i \in 2 Z} h_{i}^{2}+2 \sum_{k>0} \sum_{i \in 2 Z} h_{i} h_{i+2 k} \\
& =\left(\sum_{i \in 2 Z+1} h_{i}\right)^{2}+\left(\sum_{i \in 2 Z} h_{i}\right)^{2} .
\end{aligned}
$$

It follows from (2.3) that $\sum_{k} b_{k}=1$. Similarly, $\sum_{k} \widetilde{b}_{k}=1$.

Proposition 2.3. $M_{2 n}^{T} M_{2 n}, M_{2 n} M_{2 n}^{T}, \widetilde{M}_{2 n}^{T} \widetilde{M}_{2 n}, \widetilde{M}_{2 n} \widetilde{M}_{2 n}^{T}$ have the same eigenvalues.

Proof. Note that $M_{2 n}^{T} M_{2 n}$ and $\widetilde{M}_{2 n}^{T} \widetilde{M}_{2 n}$ have the same eigenvalues (cf. [12, Section 2]). The proof of the others is trivial.

Proposition 2.4. Assume that both $M_{2 n}=\left[\begin{array}{c}H \\ G\end{array}\right]$ and $\widetilde{M}_{2 n}=\left[\begin{array}{c}\widetilde{H} \\ \widetilde{G}\end{array}\right]$ are 2-circular matrices generated by $\{h, g\}$ and $\{\widetilde{h}, \widetilde{g}\}$, respectively, where $H, G, \widetilde{H}, \widetilde{G}$ are all $n \times 2 n$ 2-circular matrices and $n$ is large enough. Then

(1) $H H^{T}, H G^{T}, G H^{T}, G G^{T}, \widetilde{H} \widetilde{H}^{T}, \widetilde{H} \widetilde{G}^{T}, \widetilde{G} \widetilde{H}^{T}, \widetilde{G} \widetilde{G}^{T}$ are all 1-circular matrices.

(2) $H H^{T} G G^{T}-H G^{T} G H^{T}=I_{n}$.

Proof. (1) The element at the $j$ th row and the $k$ th column in $H H^{T}$ can be written as

$$
\sum_{i} h_{i+2 j} h_{i+2 k}=\sum_{i} h_{i+2(j+1)} h_{i+2(k+1)} .
$$


Since $\sum_{i} h_{i+2(j+1)} h_{i+2(k+1)}$ is the element at the $(j+1)$ th row and the $(k+1)$ th column in $H H^{T}$, it is easy to see that $H H^{T}$ is a 1-circular matrix. The proof of the others is similar.

(2) Note that $\left(M_{2 n}^{T} M_{2 n}\right)\left(\widetilde{M}_{2 n}^{T} \widetilde{M}_{2 n}\right)=I_{n}$. It implies

$$
\left(H H^{T}\right)\left(\widetilde{H} \widetilde{H}^{T}\right)+\left(H G^{T}\right)\left(\widetilde{G} \widetilde{H}^{T}\right)=I_{n}
$$

and

$$
\left(H H^{T}\right)\left(\widetilde{H} \widetilde{G}^{T}\right)+\left(H G^{T}\right)\left(\widetilde{G} \widetilde{G}^{T}\right)=O_{n},
$$

where $O_{n}$ denotes an $n \times n$ zero matrix.

Firstly, we verify that

$$
G G^{T}=\widetilde{H} \widetilde{H}^{T}, H H^{T}=\widetilde{G} \widetilde{G}^{T}, H G^{T}=-\widetilde{G} \widetilde{H}^{T}, \widetilde{H} \widetilde{G}^{T}=-G H^{T} .
$$

In fact, the element in $G G^{T}$ can be written as

$$
\begin{aligned}
\sum_{i} g_{i+2 j} g_{i+2 k} & =\sum_{i}(-1)^{1-i-2 j} \widetilde{h}_{1-i-2 j}(-1)^{1-i-2 k} \widetilde{h}_{1-i-2 k} \\
& =\sum_{i} \widetilde{h}_{i-2 j} \widetilde{h}_{i-2 k}=\sum_{i} \widetilde{h}_{i+2 j} \widetilde{h}_{i+2 k} .
\end{aligned}
$$

It is just the element in $\widetilde{H} \widetilde{H}^{T}$ at the same position. Thus, $G G^{T}=\widetilde{H} \widetilde{H}^{T}$ holds.

Similarly, the others in (2.8) can be verified.

Note that if $A$ and $B$ are 1-circular matrices, then $A B$ and $A \pm B$ are 1circular matrices, moreover, $A B=B A$ (cf. [5, Section 2]).

It follows from (2.11) and (2.12) that

$$
\begin{aligned}
O_{n} & =\left(H H^{T}\right)\left(\widetilde{H} \widetilde{G}^{T}\right)+\left(H G^{T}\right)\left(\widetilde{G} \widetilde{G}^{T}\right)=\left(H H^{T}\right)\left(\widetilde{H} \widetilde{G}^{T}\right)+\left(\widetilde{G} \widetilde{G}^{T}\right)\left(H G^{T}\right) \\
& =\left(\widetilde{G} \widetilde{G}^{T}\right)\left(\widetilde{H} \widetilde{G}^{T}\right)+\left(\widetilde{G} \widetilde{G}^{T}\right)\left(-\widetilde{G} \widetilde{H}^{T}\right)=\left(\widetilde{G} \widetilde{G}^{T}\right)\left[\left(\widetilde{H} \widetilde{G}^{T}\right)-\left(\widetilde{G} \widetilde{H}^{T}\right)\right] .
\end{aligned}
$$

Since $M_{2 n} M_{2 n}^{T}$ is a positive definite matrix, and its principal minor determinants are all positive, we have $\operatorname{det}\left(H H^{T}\right)=\operatorname{det}\left(\widetilde{G} \widetilde{G}^{T}\right)>0$, thus, $\widetilde{G} \widetilde{H}^{T}=$ $\widetilde{H} \widetilde{G}^{T}$.

Finally, by (2.10) and (2.12), we have

$$
\begin{aligned}
\left(H H^{T}\right)\left(G G^{T}\right)-\left(H G^{T}\right)\left(G H^{T}\right) & =\left(H H^{T}\right)\left(\widetilde{H} \widetilde{H}^{T}\right)+\left(H G^{T}\right)\left(\widetilde{H} \widetilde{G}^{T}\right) \\
& =\left(H H^{T}\right)\left(\widetilde{H} \widetilde{H}^{T}\right)+\left(H G^{T}\right)\left(\widetilde{G} \widetilde{H}^{T}\right)=I_{n} .
\end{aligned}
$$

The proof is completed.

We are ready to prove Theorem 2.1.

Proof of Theorem 2.1. The characteristic polynomial of $M_{2 n} M_{2 n}^{T}$ is

$$
p(\lambda)=\operatorname{det}\left(M_{2 n} M_{2 n}^{T}-\lambda I_{2 n}\right)=\operatorname{det}\left(\begin{array}{cc}
H H^{T}-\lambda I_{n} & H G^{T} \\
G H^{T} & G G^{T}-\lambda I_{n}
\end{array}\right) .
$$

Note that if $A C=C A$, then

$$
\operatorname{det}\left[\begin{array}{ll}
A & B \\
C & D
\end{array}\right]=\operatorname{det}(A D-C B)
$$


which is a simple result in linear algebra.

Thus,

$$
\begin{aligned}
p(\lambda) & =\operatorname{det}\left(\left(H H^{T}-\lambda I_{n}\right)\left(G G^{T}-\lambda I_{n}\right)-G H^{T} H G^{T}\right) \\
& =\operatorname{det}\left(\lambda^{2} I_{n}-\lambda\left(H H^{T}+G G^{T}\right)+H H^{T} G G^{T}-G H^{T} H G^{T}\right) .
\end{aligned}
$$

By Proposition 2.4, we have

$$
p(\lambda)=\operatorname{det}\left(\lambda^{2} I_{n}-\lambda\left(H H^{T}+G G^{T}\right)+I_{n}\right) .
$$

It is simple to verify that $H H^{T}, \widetilde{H} \widetilde{H}^{T}$ are $n \times n$ 1-circular matrices generated by $\left(b_{0}, b_{1}, \ldots, b_{d_{1}}, 0, \ldots, 0, b_{d_{1}}, \ldots, b_{1}\right)$ and $\left(\widetilde{b}_{0}, \widetilde{b}_{1}, \ldots, \widetilde{b}_{d_{2}}, 0, \ldots, 0, \widetilde{b}_{d_{2}}, \ldots, \widetilde{b}_{1}\right)$, respectively, where $b_{k}$ and $\widetilde{b}_{k}$ are defined in (2.5).

Note that $\lambda^{2} I_{n}-\lambda\left(H H^{T}+G G^{T}\right)+I_{n}$ is also a 1-circular matrix. We define (2.14) $f(x)=a_{1}+a_{2} x+a_{3} x^{2}+\cdots+a_{d+1} x^{d}+a_{d+1} x^{n-d}+\cdots+a_{2} x^{n-1}$, where $a_{1}=\lambda^{2}-\left(b_{0}+\widetilde{b}_{0}\right) \lambda+1, a_{i+1}=-\left(b_{i}+\widetilde{b}_{i}\right) \lambda(1 \leq i \leq d)$.

By the property of 1-circular matrix determinant in [5], we have

$$
p(\lambda)= \begin{cases}f(1) \prod_{j=1}^{r}\left|f\left(\omega^{j}\right)\right|^{2} & n=2 r+1 \\ f(1) f(-1) \prod_{j=1}^{r}\left|f\left(\omega^{j}\right)\right|^{2} & n=2 r+2\end{cases}
$$

where $\omega=\cos \left(\frac{2 \pi}{n}\right)+i \sin \left(\frac{2 \pi}{n}\right)$.

Since

$$
f(1)=\lambda^{2}-\lambda\left(\left(b_{0}+\widetilde{b}_{0}\right)+2 \sum_{i=1}^{d}\left(b_{i}+\widetilde{b}_{i}\right)\right)+1=\lambda^{2}-\lambda\left(\sum_{i}\left(b_{i}+\widetilde{b}_{i}\right)\right)+1,
$$

by Proposition 2.2, we have

$$
f(1)=\lambda^{2}-2 \lambda+1 .
$$

Using the formulas

$$
\cos \left(\frac{2 \pi(n-k) j}{n}\right)=\cos \left(2 \pi j-\frac{2 \pi k j}{n}\right)=\cos \left(\frac{2 \pi k j}{n}\right)
$$

and

we have

$$
\sin \left(\frac{2 \pi(n-k) j}{n}\right)=\sin \left(2 \pi j-\frac{2 \pi k j}{n}\right)=-\sin \left(\frac{2 \pi k j}{n}\right),
$$

$$
\begin{aligned}
& \left|f\left(\omega^{j}\right)\right|^{2} \\
= & \left|a_{1}+a_{2} \omega^{j}+a_{3} \omega^{2 j}+\cdots+a_{d+1} \omega^{d j}+a_{d+1} \omega^{(n-d) j}+\cdots+a_{2} \omega^{(n-1) j}\right|^{2} \\
= & \left(a_{1}+a_{2} \cos \left(\frac{2 \pi j}{n}\right)+\cdots+a_{d+1} \cos \left(\frac{2 \pi d j}{n}\right)+a_{d+1} \cos \left(\frac{2 \pi(n-d) j}{n}\right)+\cdots\right. \\
& \left.\quad+a_{2} \cos \left(\frac{2 \pi(n-1) j}{n}\right)\right)^{2}
\end{aligned}
$$




$$
\begin{aligned}
+ & \left(a_{2} \sin \left(\frac{2 \pi j}{n}\right)+\cdots+a_{d+1} \sin \left(\frac{2 \pi d j}{n}\right)+a_{d+1} \sin \left(\frac{2 \pi(n-d) j}{n}\right)+\cdots\right. \\
& \left.\quad+a_{2} \sin \left(\frac{2 \pi(n-1) j}{n}\right)\right)^{2} \\
= & \left(a_{1}+2 a_{2} \cos \left(\frac{2 \pi j}{n}\right)+\cdots+2 a_{d+1} \cos \left(\frac{2 \pi d j}{n}\right)\right)^{2} \\
= & \left(\lambda^{2}-\lambda\left(\left(b_{0}+\widetilde{b}_{0}\right)+2 \sum_{i=1}^{d}\left(b_{i}+\widetilde{b}_{i}\right) \cos \left(\frac{2 \pi i j}{n}\right)\right)+1\right)^{2},
\end{aligned}
$$

i.e.,

$$
\left|f\left(\omega^{j}\right)\right|^{2}=\left(\lambda^{2}-\lambda\left(\left(b_{0}+\widetilde{b}_{0}\right)+2 \sum_{i=1}^{d}\left(b_{i}+\widetilde{b}_{i}\right) \cos \left(\frac{2 \pi i j}{n}\right)\right)+1\right)^{2},
$$

where $u_{n, j}$ is defined by $(2.6)$.

If $n=2 r+2$, let $x=-1$ in (2.14), then

$$
f(-1)=\lambda^{2}-\lambda\left(\left(b_{0}+\widetilde{b}_{0}\right)+2 \sum_{i=1}^{d}(-1)^{i}\left(b_{i}+\widetilde{b}_{i}\right)\right)+1=\lambda^{2}-u_{0} \lambda+1
$$

where $u_{0}$ is defined by (2.7). The proof is completed.

Definition 2.5. Let $M$ be an $n \times n$ matrix with real elements, and $\lambda(M)$ be all eigenvalues of $M M^{T}$. Then $\lambda(M)$ is called the spectrum of matrix $M$, and $\rho(M)=\max _{i}\left|\lambda_{i}\right|$ is called the spectral radius of $M$.

Theorem 2.6. $\rho\left(M_{2 n}\right) \rightarrow \beta(n \rightarrow+\infty)$, where $\beta$ is a finite positive real number.

Proof. Define

$$
g(x)=\left(b_{0}+\widetilde{b}_{0}\right)+2\left(b_{1}+\widetilde{b}_{1}\right) \cos x+\cdots+2\left(b_{d}+\widetilde{b}_{d}\right) \cos (d x), x \in[0, \pi] .
$$

By (2.6), $g(x)$ is a continuous function in $[0, \pi]$. Therefore, there exists an $x_{0} \in[0, \pi]$ such that

$$
g\left(x_{0}\right)=\max _{x \in[0, \pi]}\{g(x)\}
$$

Define

$$
A_{n}=\left\{\frac{2 \pi j}{n}, j=1,2, \ldots,[n / 2]\right\} .
$$

For $x_{0} \in[0, \pi]$, there exists an $x_{n}=\frac{2 \pi j_{n}}{n} \in A_{n}$ such that when $n \rightarrow+\infty$, $x_{n} \rightarrow x_{0}$. Thus,

$$
g\left(x_{n}\right) \rightarrow g\left(x_{0}\right)(n \rightarrow+\infty)
$$


Note that $u_{n, j_{n}}=g\left(\frac{2 \pi j_{n}}{n}\right), \frac{\sqrt{x^{2}-4}}{2}(x>2)$ is a monotonously increasing function, and

$$
\frac{u_{n, j_{n}}+\sqrt{u_{n, j_{n}}^{2}-4}}{2} \leq \rho\left(M_{2 n}\right) \leq \frac{g\left(x_{0}\right)+\sqrt{g^{2}\left(x_{0}\right)-4}}{2}=\beta .
$$

This leads to $\rho\left(M_{2 n}\right) \rightarrow \beta(n \rightarrow+\infty)$. The proof of this theorem is completed.

Definition 2.7. $\beta=\lim _{n \rightarrow+\infty} \rho\left(M_{2 n}\right)$ is called the spectral radius of a biorthogonal wavelet.

Now we go back to (1.5) and (1.12). By Theorem 2.6, we can obtain the following theorem directly.

Theorem 2.8. Let $c^{0}=\left(c_{n}^{0}\right)_{n \in Z} \in l^{2}, c^{1}=\left(\ldots, c_{1}^{1}, c_{2}^{1}, \ldots, c_{n}^{1}, \ldots, d_{1}^{1}, d_{2}^{1}, \ldots\right.$, $\left.d_{n}^{1}, \ldots\right)$. Define a operator $T: T c^{0}=c^{1}$, where $c^{0}$ and $c^{1}$ are defined by (1.5). Then

$$
\frac{1}{\sqrt{\beta}}\left\|c^{0}\right\| \leq\left\|T c^{0}\right\| \leq \sqrt{\beta}\left\|c^{0}\right\| .
$$

In the rest of this section, we will illustrate our results by an example. The well-known 9-7 wavelet, which was studied thoroughly by Cohen et al. [1], is denoted as CDF 9-7. In this example, $d=4$, numerical computation gives

$$
\begin{aligned}
& \lambda\left(M_{18}\right)=\{0.7720,0.7720,0.8561,0.8561,0.8980,0.8980,0.9545,0.9545,1,1, \\
&1.0477,1.0477,1.1136,1.1136,1.1681,1.1681,1.2953,1.2953\}, \\
& \lambda\left(M_{20}\right)=\{0.7567,0.8025,0.8025,0.8751,0.8751,0.9053,0.9053,0.9617, \\
& 0.9617,1,1,1.0399,1.0399,1.1045,1.1045,1.1427,1.1427,1.2460, \\
&1.2460,1.3216\} .
\end{aligned}
$$

We can also calculate that

$$
\begin{aligned}
& b_{0}+\widetilde{b}_{0} \approx 2.0234, b_{1}+\widetilde{b}_{1} \approx-0.0159, b_{2}+\widetilde{b}_{2} \approx 0.0064, b_{3}+\widetilde{b}_{3} \approx-0.0036, \\
& b_{4}+\widetilde{b}_{4} \approx 0.0014, \text { and } u_{n, j} \rightarrow u(u \approx 2.078)(j=[n / 2], n \rightarrow+\infty) .
\end{aligned}
$$

Thus, $\beta \approx 1.3216$.

\section{A design method based on minimizing spectral radius}

Define

$$
\begin{aligned}
& m_{0}(\xi)=2^{-1 / 2} \sum_{n} h_{n} e^{-i n \xi} \\
& \widetilde{m}_{0}(\xi)=2^{-1 / 2} \sum_{n} \widetilde{h}_{n} e^{-i n \xi}
\end{aligned}
$$

Assume that (3.1) can be factored as

$$
\begin{aligned}
& m_{0}(\xi)=\left(\frac{1+e^{-i \xi}}{2}\right)^{L} F(\xi), \\
& \widetilde{m}_{0}(\xi)=\left(\frac{1+e^{-i \xi}}{2}\right)^{\widetilde{L}} \widetilde{F}(\xi) .
\end{aligned}
$$


The idea of classical design method for biorthogonal wavelet is to choose as high $(L, \widetilde{L})$ as possible in (3.2)(CDF9-7 was successfully designed in [1]). Applying this method to 8-8-tap wavelets, we obtain an 8-8-tap wavelet denoted as OR (8-8):

$$
\begin{aligned}
& h=\sqrt{2}(0.0534975,-0.0872258,-0.0692208,0.602949), \\
& \widetilde{h}=\sqrt{2}(-0.0228179,-0.0372038,0.133432,0.42659),
\end{aligned}
$$

(whereas the other half is symmetric and so skipped), and the highest $(L, \widetilde{L})=$ $(5,3)$. However, this wavelet is not as good as CDF9-7 in terms of image compression. Computation shows that its spectral radius is slightly large, see Table 1.

Table 1. Spectral radius

\begin{tabular}{|c|c|c|}
\hline Wavelets & spectrum radius $\beta$ & $\beta^{\frac{1}{2}}$ \\
\hline OR8-8 & 2.6432 & 1.6258 \\
\hline OP8-8 & 1.7612 & 1.3271 \\
\hline OP12-8 & 1.4714 & 1.2130 \\
\hline OP16-8 & 1.3824 & 1.1758 \\
\hline CDF9-7 & 1.3216 & 1.1496 \\
\hline
\end{tabular}

From (2.18), it is obvious that $T$ is a unitary operator if and only if $\sqrt{\beta}=1$. If $T$ is derived from a biorthogonal wavelet, then it is not a unitary operator. In order to keep the stability of transform, we hope the operator is as near a unitary one as possible, i.e., $\sqrt{\beta}$ is as near 1 as possible. Based on this idea, we can minimize the spectral radius to obtain some biorthogonal wavelets.

Now we relax the condition of the highest $\widetilde{L}$, for example, let $\widetilde{L}$ decrease to 1 from 3 in the case of 8-8-tap. Then we can use an extra degree of freedom to minimize the spectral radius.

Assume that a wavelet system contains a vector of s variables, which is represented by $X=\left(x_{1}, x_{2}, \ldots, x_{s}\right)$. Then $h, \widetilde{h}, b_{k}$ and $\widetilde{b}_{k}$ in $(2.5), u_{n, j}$ in $(2.6)$ are functions of $X$. To formulate the optimization problem, define

$$
u_{n, 0}= \begin{cases}0, & n=2 r+1 \\ u_{0}, & n=2 r+2\end{cases}
$$

where $u_{0}$ is defined by (2.7). Consider the optimization problem for a large enough $n$ as follows:

$$
u_{n}=\min _{X}\left\{\max _{0 \leq j \leq r}\left\{u_{n, j}\right\}\right\}
$$

Let $\beta_{n}=\frac{u_{n}+\sqrt{u_{n}^{2}-4}}{2}$. Then the limit of $\beta_{n}$ is the spectral radius. In fact, the optimization process stops whenever $\left|\beta_{n+1}-\beta_{n}\right|<\varepsilon$, where $\varepsilon>0$ is given. We take $\varepsilon=10^{-5}$ for numerical computations in this paper. Generally speaking, $n$ has to be larger than 400. Obviously, the optimization process is very complex and only approximate solutions can be obtained. 
Applying the same process to 8-8-tap, 12-8-tap and 16-8-tap wavelets, we obtained three optimal wavelets with other half symmetric part skipped as before.

OP8-8:

$h \approx \sqrt{2}(0.10588478,-0.21250827,0.13072889,0.47589460)$,

$\widetilde{h} \approx \sqrt{2}(-0.03146955,-0.06315864,0.12478045,0.46984774)$, with $(L, \widetilde{L})=(5,1)$.

OP12-8:

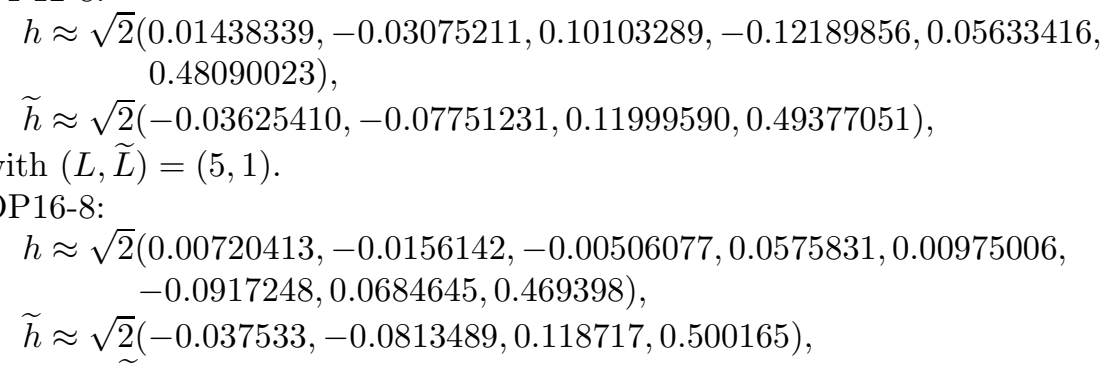
with $(L, \widetilde{L})=(5,3)$. See Fig. 1 for the graphs of the scaling functions and wavelets in this example.
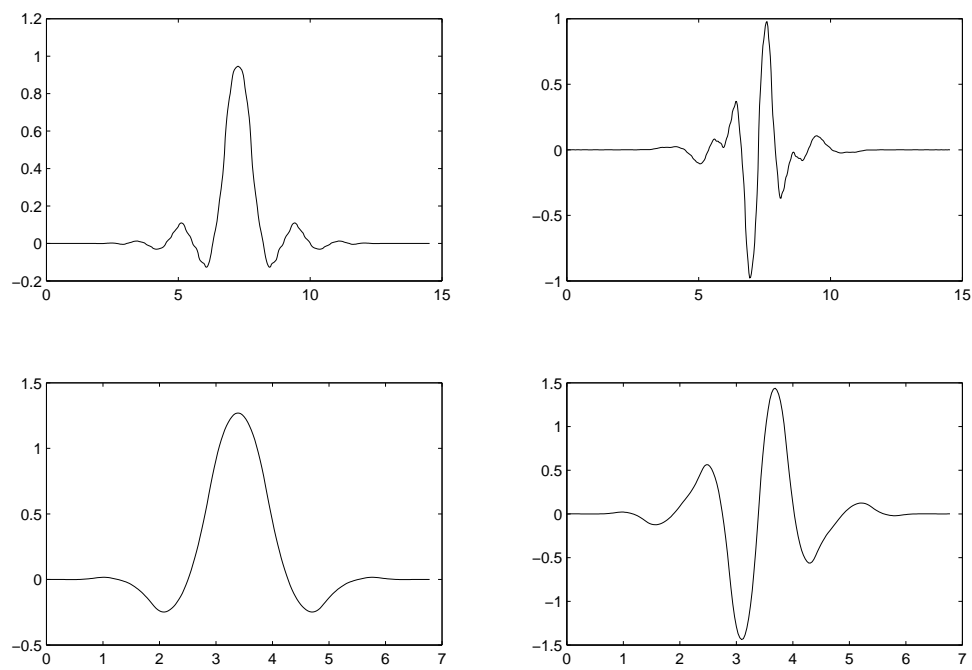

Fig. 1. OP16-8: $\phi, \widetilde{\phi}$ a dual scale functions; $\psi, \widetilde{\psi}$ a dual wavelet functions.

In order to test the performance of these wavelets, we apply the SPIHT algorithms [7] to the experiments of image compression coding. In these experiments, we use the typical and standard test images such as Lena and Barbara, 
which are decomposed 5 levels, with the Huffman entropy coding technologies. The PSNR $(\mathrm{dB})$ is used to evaluate the quality of decoded images. From the test results in Table 2 and Table 3, we can see that the OP16-8 has significant advantage in image compression coding. In fact, OP16-8 is superior for almost all test images, with further details of test skipped due to space limit.

Note that, in general, smaller norm does not necessarily make the performance better. A counter example is Haar wavelet with obviously poor performance. As the optimization procedure is applied to the wavelet systems with even lengths filters, the smallest spectral radius must be 1 if no other constraints imposed on the filters. Usually, the high vanishing moments are necessary.

Table 2. Test results for Lena

\begin{tabular}{|c|c|c|c|c|}
\hline & $1: 4$ & $1: 8$ & $1: 16$ & $1: 32$ \\
\hline OR8-8 & 43.51 & 39.24 & 36.06 & 32.79 \\
\hline OP8-8 & 43.97 & 39.53 & 36.33 & 33.10 \\
\hline OP12-8 & 44.33 & 39.82 & 36.53 & 33.35 \\
\hline OP16-8 & 44.73 & 40.11 & 36.81 & 33.58 \\
\hline CDF9-7 & 44.52 & 40.03 & 36.78 & 33.54 \\
\hline
\end{tabular}

Table 3. Test results for Barbara

\begin{tabular}{|c|c|c|c|c|}
\hline & $1: 4$ & $1: 8$ & $1: 16$ & $1: 32$ \\
\hline OR8-8 & 41.35 & 34.90 & 29.64 & 25.69 \\
\hline OP8-8 & 41.61 & 35.51 & 30.24 & 26.51 \\
\hline OP12-8 & 41.78 & 35.76 & 30.60 & 26.87 \\
\hline OP16-8 & 42.44 & 36.45 & 31.38 & 27.45 \\
\hline CDF9-7 & 42.22 & 36.01 & 30.99 & 27.20 \\
\hline
\end{tabular}

\section{Conclusions}

Algebraic methods are often effective in studying the wavelet problems. In this paper, an important index of spectral radius for biorthogonal wavelet is proposed based on the eigenvalue-method. It provides us another viewpoint to examine and evaluate the wavelets. The circular matrix theory is no doubt an effective tool in wavelet analysis. The concepts, results and method proposed in this paper could be helpful for the estimate of bounds of wavelet frames.

Acknowledgments. The authors would like to thank the editors and reviewers for their valuable comments, which greatly improved the readability of this paper.

\section{References}

[1] A. Cohen, I. Daubechies, and J. C. Feauveau, Biorthogonal bases of compactly supported wavelets, Commu. Pure Appl. Math. 45 (1992), no. 5, 485-560.

[2] I. Daubechies, Orthonormal bases of compactly supported wavelets, Comm. Pure Appl. Math. 41 (1988), no. 7, 909-996.

[3] _ , Ten Lectures on Wavelets, Philadelphia, SIAM Pub., 1992. 
[4] H. O. Kim, R. Y. Kim, Y. J. Lee, and J. Yoon, Quasi-interpolatory refinable functions and construction of biorthogonal wavelet systems, Adv. Comput. Math. 33 (2010), no. $3,255-283$.

[5] T. L. Li, Characters of circular matrix, Science Bulletin in Chinese 2 (1982), 30-33.

[6] T. Q. Nguyen and P. P. Vaidyanathan, Two-channel perfect reconstruction FIR QMF structures which yield linear-phase analysis and synthesis filters, IEEE Trans. Acoustics Speech Signal Process. 37 (1989), 676-690.

[7] A. Said and W. A. Pearlman, A new, fast and efficient image codec based on set partitioning in hierarchical trees, IEEE Trans. Circuits System Video Tech. 6 (1996), 243-250.

[8] W. Sweldens, The lifting scheme: a constructing of second generation wavelet, SIAM. Math. Anal. 29 (1998), no. 2, 511-546.

[9] M. Vetterli and D. L. Gall, Perfect reconstruction FIR filter banks, some properties and factorizations, IEEE Trans. Acoust. Speech, Signal Process. 37 (1989), no. 7, 1057-1071.

[10] G. Q. Wang, Matrix methods of constructing wavelet filters and discrete hyper-wavelet transforms, Opt. Eng. 39 (2000), no. 4, 1080-1087.

[11] G. Q. Wang and W. W. Yuan, Generic 9-7 tap wavelets filters and their performance studies on image compression, Acta. Electron. Sin. 29 (2001), 130-132.

[12] _ Optimal model for biorthogonal wavelet filters, Opt. Eng. 42 (2003), no. 2, $350-356$.

QINGYUN ZOU

Department of Mathematics

Hunan University of Arts and Science

Changde, 415000, Hunan, P. R. China

AND

College of Mathematics and Computer Science

HUNAN NORMAL UNIVERSITY

Changsha, 410081, Hunan, P. R. China

E-mail address: qyzou2000@sina.com

GuOQIU Wang

College of Mathematics and Computer Science

HUNAN NORMAL UNIVERSITY

Changsha, 410081, Hunan, P. R. China

E-mail address: gqwang1028@sina.com

Mengyun Yang

Department of Science and Information Science

SHAOYANG UNIVERSITY

Shaoyang, 422000, Hunan, P. R. China

E-mail address: yanginter1@126.com 